\title{
Aspetti epidemiologici della calcolosi di calcio in Italia: distribuzione dei fenotipi intermedi nella popolazione italiana
}

\author{
T. Arcidiacono', F. Rainone', A. Terranegra ${ }^{2}$, E. Dogliotti ${ }^{2}$, V. Paloschi', \\ G. Lauriero' ${ }^{1}$ A. Aloia ${ }^{2}$, L. Soldati², G. Vezzoli ${ }^{1}$ \\ ${ }^{1}$ Unità di Nefrologia e Dialisi, Istituto Scientifico San Raffaele, Università Vita Salute, Milano \\ ${ }^{2}$ Dipartimento di Scienze e Tecnologie Biomediche, Università degli Studi di Milano, Milano
}

\begin{abstract}
Questo lavoro è stato scritto a nome di tutti i membri del GENIAL network che devono essere considerati come coautori. Membri del GENIAL network: Donatella Spotti ${ }^{1}$, Daniele Cusi ${ }^{2}$, Loris Borghi ${ }^{3}$, Angela Guerra ${ }^{3}$, Franca Allegri ${ }^{3}$, Beatrice Prati ${ }^{3}$, Tiziana Meschi ${ }^{3}$, Antonio Nouvenne ${ }^{3}$, Giovanni Gambaro $^{4}$, Antonio Lupo ${ }^{5}$, Antonia Fabris ${ }^{5}$, Naveed Aslam ${ }^{5}$, Domenico Rendina ${ }^{6}$, Giuseppe Mossetti ${ }^{6}$, Giampaolo De Filippo ${ }^{6}$, Pasquale Strazzullo ${ }^{6}$, Maria Luisa Brandi $^{7}$, Emanuele Croppi ${ }^{7}$, Annalisa Tanini ${ }^{7}$, Alberto Falchetti ${ }^{7}$, Alessia Gozzini ${ }^{7}$, Alberto Trinchieri ${ }^{8}$, Renata Caudarella ${ }^{9}$, Adamasco Cupisti ${ }^{10}$, Lorenzo Citron ${ }^{11}$, Franca Anglani ${ }^{11}$, Angela D’Angelo ${ }^{11}$, Piera Bellinzoni ${ }^{12}$, Fabio Vescini ${ }^{13}$
\end{abstract}

${ }^{1}$ Unità di Nefrologia e Dialisi, Istituto Scientifico San Raffaele, Università Vita Salute, Milano

${ }^{2}$ Dipartimento di Scienze e Tecnologie Biomediche, Sezione di Nefrologia, Università degli Studi di Milano, Milano

${ }^{3}$ Dipartimento di Scienze Cliniche, Università di Parma, Parma

${ }^{4}$ Divisione di Nefrologia e Dialisi dell'Ospedale Universitario Colombo-Gemelli, Università Cattolica, Scuola di Medicina, Roma

${ }^{5}$ Divisione di Nefrologia, Dipartimento di Scienze Chirurgiche e Biomediche, Ospedale Universitario di Verona

${ }^{6}$ Dipartimento di Medicina Clinica e Sperimentale, Università "Federico II", Napoli

${ }^{7}$ Dipartimento di Medicina Interna, Università di Firenze, Firenze

${ }^{8}$ Unità di Urologia, Ospedale A. Manzoni, Lecco

${ }^{9}$ Villa Maria-Fondazione di Scienze della Salute, Lugo, Ravenna

${ }^{10}$ Unità di Nefrologia, Dipartimento di Medicina Interna, Università di Pisa, Pisa

${ }^{11}$ Unità di Nefrologia, Laboratorio di Istomorfologia Renale e di Biologia Molecolare, Dipartimento di Scienze Mediche e Chirurgiche, Università di Padova

${ }^{12}$ Unità di Urologia e Dialisi, Istituto Scientifico San Raffaele-Ospedale Turro, Milano

${ }^{13}$ Unità di Metabolismo Osseo, Ospedale di Gorizia, Gorizia

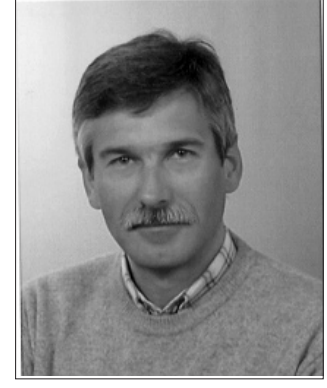

G. Vezzoli

\section{Introduzione}

La calcolosi renale di calcio è una malattia che interessa il 5-10\% della popolazione italiana (1). E generalmente riconosciuto che difetti come l'ipercalciuria, l'iperuricuria, l'iperossaluria e l'ipocitraturia compaiono con frequenza significativamente maggiore nei pazienti con la calcolosi di calcio rispetto alla popolazione normale, ma la loro distribuzione varia nelle diverse casistiche $(2,3)$. Questi difetti sono stati oggetto di speculazione circa il loro coinvolgimento patogenetico nella calcolosi che rimane però ancora poco chiaro, anche se si ammette che il loro contributo alla litogenesi potrebbe realizzarsi attraverso la modificazione della soprassaturazione delle urine per dei sali litogeni $(4,5)$. Anche altri difetti urinari sono stati registrati nei pazienti con calcolosi renale calcica e potrebbero avere un'importanza patogenetica, almeno in una parte dei casi. L'elevata escrezione di sodio e fosfato, con conseguente ipofosforemia, e la ridotta escrezione di potassio sono spesso presenti nei pazienti e potrebbero essere dovuti ad abituduni dietetiche particolari (6), ma il loro significato è stato poco approfondito nella letteratura medica.

Lo studio dei difetti urinari e delle relazioni tra questi difetti nei pazienti calcolotici potrebbe essere uno strumento per identificare sottogruppi di pazienti simili da un punto di vista metabolico, nutrizionale o genetico, che condividono fattori di rischio litogeno. Nonostante questa possibilità, gli studi che analizzano le caratteristiche fenotipiche dei pazienti con calcolosi renale si sono limitati a studiare le frequenze dei singoli difetti tralasciando di verificare le interrelazioni tra gli stessi e il peso dei singoli difetti nello sviluppo della calcolosi. Abbiamo perciò analizzato una popolazione italiana di 


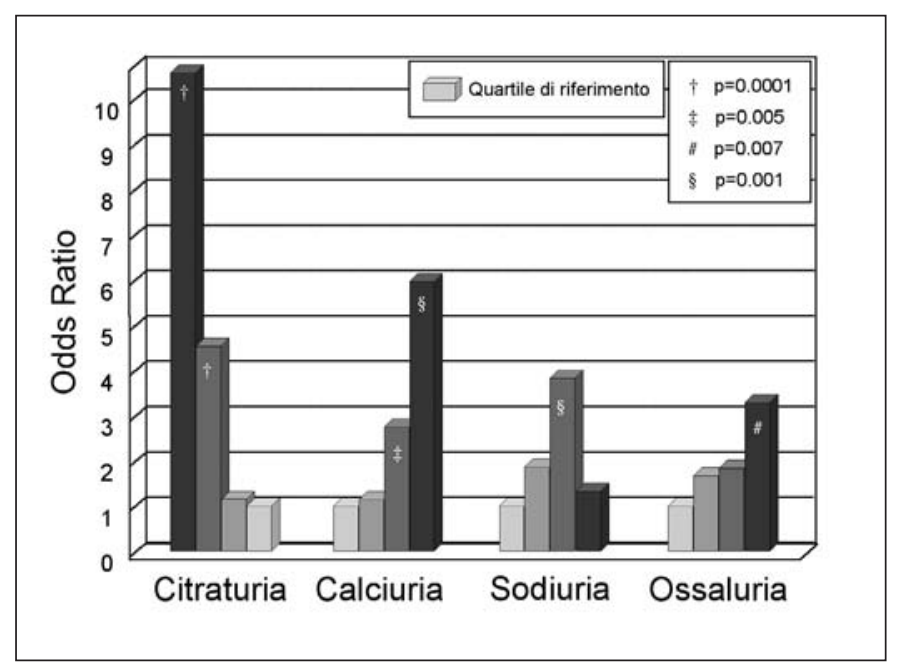

Fig. 1 - Nella popolazione studiata, il rischio di calcolosi renale di calcio (espresso come Odds Ratio) è associato alla escrezione di citrato, calcio, sodio e ossalato nelle urine di 24 ore, espresse in quartili. Il rischio è aumentato nel primo e secondo quartile di citraturia, nel terzo e quarto quartile di calciuria, nel quarto quartile di ossaluria e nel terzo quartile di sodiuria.

pazienti affetti da calcolosi di calcio per definire la distribuzione dei difetti urinari e il loro contributo al rischio di calcolosi.

\section{Metodi}

Abbiamo studiato 797 pazienti affetti da calcolosi renale idiopatica (445 uomini e 352 donne, età $46 \pm 14.2$ anni) paragonandoli con 164 controlli (74 uomini e 70 donne, età $45 \pm 12.5$ anni). I pazienti sono stati reclutati presso gli Ospedali S. Orsola di Bologna, Careggi di Firenze, Manzoni di Lecco, Policlinici di Milano, Napoli, Padova Pisa e Verona. I pazienti non erano affetti da altre patologie oltre alla calcolosi e non assumevano farmaci. Forme secondarie della medesima erano escluse, mediante dosaggio di calcemia, $\mathrm{pH}$ urine ed elettroliti plasmatici. La diagnosi di calcolosi di calcio era posta mediante analisi della composizione del calcolo oppure mediante riscontro della sua radio-opacità.

Ipercalciuria è stata definita come l'escrezione di calcio superiore a $300 \mathrm{mg} / 24 \mathrm{~h}$ negli uomini oppure $250 \mathrm{mg} / 24$ $\mathrm{h}$ nelle donne oppure superiore a $4 \mathrm{mg} / \mathrm{kg}$ di peso nelle $24 \mathrm{~h}$ per entrambi i sessi. Ipocitraturia è stata definita l'escrezione di citrato minore di $350 \mathrm{mg} / 24 \mathrm{~h}$. Iperuricuria è stata definita l'escrezione di urato maggiore di 700 $\mathrm{mg} / 24 \mathrm{~h}$. Iperossaluria è stata considerata l'escrezione di ossalato maggiore di $40 \mathrm{mg} / 24 \mathrm{~h}$. Ipersodiuria è stata definita l'escrezione di sodio maggiore di $3000 \mathrm{mmol} / 24$ h. Il limite per definire l'iperescrezione di sodio è stato scelto in modo arbitrario, mentre per le altre variabili, il valore limite ha un riscontro nella letteratura medica. Le variabili quantitative sono state espresse come media \pm deviazione standard e sono state confrontate mediante test di Student o ANOVA ad una via con test di Tukey per i confronti tra gruppi. La distribuzione delle variabili qualitative sono state confrontate mediante test chi-quadrato. Un modello di regressione logistica multinomiale è stato valutato considerando la calcolosi renale come variabile dipendente e fosforemia, paratormonemia, sodiuria, potassiuria, calciuria, fosfaturia, citraturia, ossalaturia, uricuria (ciascuna divisa in quartili) come variabili indipendenti. Età, sesso e peso corporeo sono stati considerati come cofattori nella regressione logistica. Il rischio di calcolosi è stato espresso come Odds Ratio con intervallo di confidenza al 95\% $(95 \% \mathrm{CI})$. L'analisi è stata condotta con $\alpha=0.05$ e test a due code ed è stato utilizzato il software SPSS 11 su computer MacBook Pro.

\section{Risultati}

\section{Distribuzione delle variabili}

Nei pazienti studiati è stata osservata una frequenza di ipercalciuria ( 44.9 vs $22.7 \%, \mathrm{p}=0.0001)$, ipocitraturia $(38.3$ vs $14 \%, \mathrm{p}=0.0001)$ e iperuricuria $(19.3 \%$ vs $8.8 \%, \mathrm{p}=0.005)$ maggiore nei calcolotici che nei normali. Viceversa la iperossaluria era distribuita in modo simile nei calcolotici e controlli ( $15.9 \%$ vs $10.1 \%, \mathrm{p}=0.08)$.

Tra le variabili misurate, la fosforemia $(3.19 \pm 0.651$ vs $3.41 \pm 0.589 \mathrm{mmol} / \mathrm{L}, \mathrm{p}=0.001)$, la potassiuria $(53 \pm 21.7 \mathrm{vs}$ $58 \pm 20.1 \mathrm{mmol} / 24 \mathrm{~h}, \mathrm{p}=0.04)$ e la citraturia $(485 \pm 281$ vs $635 \pm 280 \mathrm{mg} / 24 \mathrm{~h}, \mathrm{p}=0.0001$ ) erano più basse nei pazienti calcolotici rispetto ai normali, mentre sodiuria $(171 \pm 63.6$ vs $157 \pm 63.8 \mathrm{mmol} / 24 \mathrm{~h}, \mathrm{p}=0.017)$, calciuria $(262 \pm 139.6$ vs $209 \pm 112 \mathrm{mg} / 24 \mathrm{~h}, \mathrm{p}=0.0001)$ e ossaluria $(30 \pm 16.3 \mathrm{vs}$ $25 \pm 12.2 \mathrm{mg} / 24 \mathrm{~h}, \mathrm{p}=0.001)$ erano pià elevate nei primi. Il BMI $(25 \pm 4.05$ vs $24 \pm 3.63$, $p=0.37)$, la calcemia $(9.44 \pm 0.44$ vs $9.52 \pm 0.52 \mathrm{mg} / \mathrm{dL})$, la fosfaturia $(812 \pm 302$ vs $806 \pm 333.5$ $\mathrm{mg} / 24 \mathrm{~h}, \mathrm{p}=0.23)$ e l'uricuria $(531 \pm 184$ vs $516 \pm 151 \mathrm{mg} / 24$ $h, p=0.36$ ) non erano significativamente diversi nei due gruppi.

Nel modello di regressione logistica utilizzato (Fig. 1), il rischio di calcolosi era aumentato nel primo e secondo quartile di citraturia (il quartile più alto è stato usato come riferimento), nel terzo e quarto quartile di calciuria, nel quarto quartile di ossaluria (primo quartile usato come riferimento per entrambe le variabili).

L'età di esordio della calcolosi era più tardiva nei pa- 
zienti del quarto quartile di citraturia $(\mathrm{n}=117,37 \pm 13.2$ anni, $\mathrm{p}=0.006)$ e del terzo quartile $(\mathrm{n}=122,36 \pm 14.7$ anni, $\mathrm{p}=0.031$ ) rispetto ai pazienti del primo quartile di citraturia ( $\mathrm{n}=155,31 \pm 13.0$ anni) nei quali l'esordio era quindi più precoce. Nei pazienti del secondo quartile di citraturia l'esordio avveniva a $32 \pm 13.8$ anni. Non era rilevata relazione dell'età di esordio con calciuria, ossaluria, uricuria e sodiuria.

\section{Relazioni tra i difetti di escrezione}

La condizione di ipocitraturia era più frequente nei pazienti normocalciurici rispetto agli ipercalciurici, mentre iperuricuria, iperossaluria ed ipersodiuria erano più frequenti nei pazienti ipercalciurici (Tab. I). I pazienti ipocitraturici erano più spesso normocalciurici e normouricurici rispetto ai normocalciurici.

Il rischio litogeno era significativamente aumentato nei pazienti ipercalciurici e normocitraturici $(\mathrm{n}=244$, OR 2.7, 95\% CI 1.7-4.2, $\mathrm{p}=0.0001$ ) e nei pazienti ipocitraturici e normocalciurici ( $\mathrm{n}=185$, OR 4.4, 95\% CI 2.5-7.8, $\mathrm{p}=0.0001$ ), ma era più alto nei pazienti sia ipercalciurici che ipocitraturici ( $\mathrm{n}=111$, OR 8.9, 95\%CI 3.5-22-6, $\mathrm{p}=0.0001$ ).

L'età di esordio della calcolosi era più tardiva nei pazienti normocalciurici e normocitraturici $(\mathrm{n}=160,37 \pm 14.2$ anni), rispetto ai pazienti ipocitraturici e normocalciurici $(\mathrm{n}=123,31 \pm 12.6$ anni; $\mathrm{p}=0.001)$ ed a quelli ipercalciurici e ipocitraturici $(\mathrm{n}=89,32 \pm 13.9, \mathrm{p}=0.019)$. L'età di esordio nei pazienti ipercalciurici e normocitraturici $(n=158)$ era $34 \pm 13.7$ anni.

La frequenza di ipocitraturia si riduceva significativamente con l'aumentare dell'età d'insorgenza della calcolosi $(44.5 \%$ dei pazienti con esordio ad età miniore di 30 anni erano ipocitraturici, $22.2 \%$ nei pazienti con esordio oltre 60 anni, $\mathrm{p}=0.036$ ). La frequenza di iperuricuria aumentava con l'aumento dell'età di esordio, era maggiore tra 41 e 50 anni (21.9\%) per poi ridursi $(\mathrm{p}=0.035)$. Relazioni simili non erano trovate per calciuria ed ossaluria.

\section{Discussione}

Allo scopo di individuare sottogruppi omogenei di pazienti con calcolosi renale di calcio, abbiamo studiato una popolazione di calcolotici reclutati in centri specialistici italiani per valutare le relazioni tra le diverse variabili tipicamente associate alla calcolosi di calcio. I risultati ottenuti confermano i dati generali già noti di associazione tra ipercalciuria, iperuricuria, iperossaluria, ipocitraturia e calcolosi di calcio ai quali si aggiungono anche un aumento della escrezione di sodio e l'ipofosfatemia (2, 7). Alla base di questo difetto è opinione comune che vi siano molteplici fattori genetici e nutrizionali. La sodiuria trova la causa del suo aumento in motivi nutrizionali e la sua relazione con la calcolosi indica che la dieta ricca in sodio predispone alla calcolosi. Questa relazione si giustifica attraverso diversi meccanismi per mezzo dei quali l'accresciuto carico salino stimola l'escrezione di diverse sostanze tra le quali principalmente il calcio e l'acido urico (8). L’ipofosfatemia è verosimilmente secondaria ad una riduzione della soglia di escrezione del fosfato a livello renale a fronte di una normale fosfaturia.

I risultati evidenziano come l'ipocitraturia sia la variabile più determinante per lo sviluppo della calcolosi di calcio. Nella popolazione studiata essa aumenta il rischio litogeno in modo più marcato rispetto alle altre variabili

TABELLA I - FREQUENZA PERCENTUALE DI IPEROSSALURIA, IPERURICURIA, IPERSODIURIA E IPOCITRATURIA NEI PAZIENTI IPERCALCIURICI E NORMOCALCIURICI CON CALCOLOSI DI CALCIO E FREQUENZA DI IPEROSSALURIA, IPERURICURIA, IPERSODIURIA E IPERCALCIURIA NEI PAZIENTI IPOCITRATURICI E NORMOCITRATURICI

\begin{tabular}{|c|c|c|c|c|}
\hline & $\begin{array}{l}\text { Pazienti con } \\
\text { Ipercalciuria }\end{array}$ & $\begin{array}{c}\text { Pazienti con } \\
\text { Normocalciuria }\end{array}$ & $\begin{array}{l}\text { Pazienti con } \\
\text { Ipocitraturia }\end{array}$ & $\begin{array}{l}\text { Pazienti con } \\
\text { Normocitraturia }\end{array}$ \\
\hline $\mathrm{N}$ & 317 & 395 & 275 & 437 \\
\hline Ipercalciuria (\%) & & & 38.5 & $48.3^{*}$ \\
\hline Iperossaluria (\%) & 21.4 & $13.3 \dagger$ & 15.1 & 17.1 \\
\hline Iperuricuria (\%) & 20.0 & 12.5 II & 11.4 & $18.3 \ddagger$ \\
\hline Ipersodiuria (\%) & 5.6 & $1.6 \#$ & 3.8 & 3.5 \\
\hline Ipocitraturia (\%) & 33.4 & $42.8 \S$ & & \\
\hline
\end{tabular}

\footnotetext{
* $\mathrm{p}=0.011$ vs ipocitraturici

$\dagger \mathrm{p}=0.005$ vs ipercalciurici

II $\mathrm{p}=0.007$ vs ipercalciurici

$\neq \mathrm{p}=0.019$ vs ipocitraturici

\# $\mathrm{p}=0.002$ vs ipercalciurici

$\S \mathrm{p}=0.011$ vs ipercalciurici
} 
studiate. Inoltre la citraturia è l'unica variabile che appare capace di spiegare l'età di esordio della calcolosi, dato che i pazienti con valori più bassi di citraturia avevano età di esordio più precoce. La citraturia potrebbe perciò influenzare la severità della malattia oltre che favorirne l'insorgenza.

La calciuria è il difetto più frequente nella popolazione dei calcolotici, tuttavia non si correla con l'età di esordio e sembra perciò essere relativamente poco influente sulle caratteristiche cliniche della malattia calcolotica nei pazienti analizzati. Lo stesso è stato osservato per uricuria e ossaluria che però sembrano causare un rischio litogeno inferiore a quello prodotto da calciuria e citraturia. Il loro peso litogeno probabilmente aumenta in specifiche situazioni, come sembra accadere all'iperuricuria che potrebbe essere associata al rischio di calcolosi nei pazienti con esordio tra 40 e 60 anni, mentre non contribuisce al rischio di calcolosi calcica nell'intera popolazione.

L'analisi condotta ha consentito di osservare che la condizione di ipercalciuria era associata a iperuricuria, iperossaluria e ad un elevato apporto dietetico di sodio, così come dimostrato dall'elevata sodiuria. I pazienti ipercalciurici presentavano inoltre normocitraturia con maggiore frequenza rispetto ai pazienti normocalciurici. Questi risultati evidenziano che nei pazienti con calcolosi renale l'escrezione di calcio, urato e ossalato tendono ad aumentare insieme. È verosimile che l'aumentato apporto di sodio contribuisca a stimolare l'escrezione di queste sostanze, anche se un substrato genetico particolare potrebbe rendere il tubulo di questi pazienti più sensibili al carico salino. Questi pazienti possono rappresentare un gruppo omogeneo per caratteristiche funzionali, nutrizionali e genetiche dalle quali sarebbe esclusa la citraturia. Un secondo gruppo di pazienti potrebbe viceversa essere caratterizzato dalla presenza di ipocitraturia senza ulteriori fattori, dato che la ipocitraturia è più frequente tra i pazienti normolcalciurici e normouricurici ed è capace da sola di spiegare l'età di esordio e lo sviluppo di calcoli interferendo in modo moltiplicativo con l'ipercalciuria. Ovviamente, se questa ipotesi fosse vera, come per tutti i caratteri complessi dovremmo aspettarci che i pazienti dei due gruppi non siano completamente separati l'uno dall'altro, ma che esista un ampio gruppo di pazienti nei quali le caratteristiche dei due gruppi si sovrappongono, rendendo difficile l'attribuzione ad uno o all'altro gruppo.

Tutti questi risultati indicano che per evitare fattori confondenti, negli studi patogenetici, nutrizionali o genetici sulla calcolosi renale di calcio dovremo distinguere i pazienti ipercalciurici dai pazienti ipocitraturici. In altre parole dovremo distinguere i pazienti "iperescretori” da quelli "ipocitraturici”, nei quali un diverso meccanismo patogenetico potrebbe essere attivo. Nel primo gruppo i promotori della precipitazione salina potrebbero giocare un ruolo patogenetico principale, mentre nel secondo gruppo l'inibizione della precipitazione esercitata dal citrato potrebbe venir meno.

\author{
Indirizzo degli Autori: \\ Giuseppe Vezzoli, MD \\ Unità di Nefrologia e Dialisi \\ IRCCS Ospedale San Raffaele \\ via Olgettina, 60 \\ 20132 Milano, Italy \\ vezzoli.giuseppe@hsr.it
}

\section{Bibliografia}

1. Trinchieri A, Coppi F, Montanari E, Del Nero A, Zanetti $\mathrm{G}$, Pisani E. Increase in the prevalence of symptomatic upper urinary tract stones during the last ten years. Eur Urol 2000, 37: 23-5.

2. Churan GC, Taylor EN. 24-h uric acid excretion and the risk of kidney stones. Kidney Int 2008; 73: 489-96.

3. Levy FL, Adams-Huet B, Pak CYC. Ambulatory evaluation of nephrolithiasis: an update of a 1980 protocol. Am J Med 1995; 98: 50-9.

4. Sakhaee K. Recent advances in the pathophysiology of nephrolithiasis. Kidney Int 2009, 75: 585-95.
5. Kavanagh JP. Supersaturation and renal precipitation: the key to stone formation? Urol Res 2006; 34: 81-5.

6. Curhan GC, Willett WC, Rimm EB, Stampfer MJ. A prospective study of dietary calcium and other nutrients and the risk of symptomatic kidney stones. N Engl J Med 1993; 328: 833-8.

7. Prie D, Ravery V, Boccon-Gibod L, Friedlander G. Frequency of renal phosphate keak among patients with nephrolithiasis. Kidney Int 2001; 60: 272-6.

8. Phillips MJ, Cooke JN. Relation between urinary calcium and sodium in patients with idiopathic hypercalciuria. Lancet 1967; 1: 1354-7. 